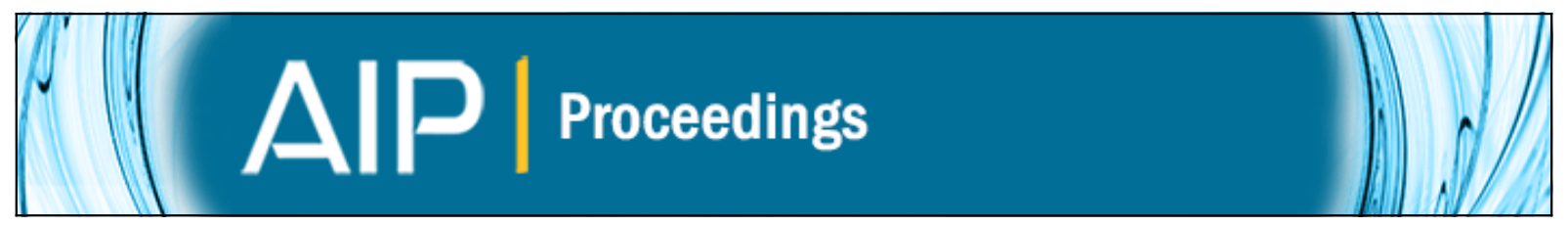

Contrast sensitivity test and conventional and high frequency audiometry: information beyond that required to prescribe lenses and headsets

S. A. Comastri, G. Martin, J. M. Simon, C. Angarano, S. Dominguez, F. Luzzi, M. Lanusse, M. V. Ranieri, and C. M. Boccio

Citation: AIP Conference Proceedings 992, 63 (2008); doi: 10.1063/1.2926943

View online: http://dx.doi.org/10.1063/1.2926943

View Table of Contents: http://scitation.aip.org/content/aip/proceeding/aipcp/992?ver=pdfcov

Published by the AIP Publishing

Articles you may be interested in

Note: A multifunction lens test system for imaging quality evaluation

Rev. Sci. Instrum. 81, 016105 (2010); 10.1063/1.3280162

Measurement system of the refractive power of spherical and sphero-cylindrical lenses with the magnification ellipse fitting method

Rev. Sci. Instrum. 80, 115109 (2009); 10.1063/1.3262503

Inspection of refractive $\mathrm{x}$-ray lenses using high-resolution differential phase contrast imaging with a microfocus $\mathrm{x}$ ray source

Rev. Sci. Instrum. 78, 093707 (2007); 10.1063/1.2786273

X-ray parabolic lenses made from glassy carbon by means of laser

Rev. Sci. Instrum. 77, 063113 (2006); 10.1063/1.2198791

Simulation and testing of a graded negative index of refraction lens

Appl. Phys. Lett. 87, 091114 (2005); 10.1063/1.2037202 


\title{
Contrast sensitivity test and conventional and high frequency audiometry: information beyond that required to prescribe lenses and headsets
}

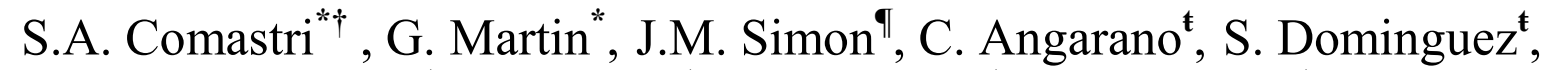

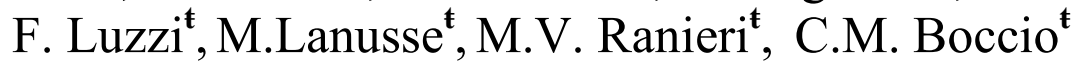 \\ "Grupo Óptica y Visión, Fac. Ingeniería, Universidad Buenos Aires, Colón 850, C1063ACV, Bs.As. Argentina \\ ${ }^{\dagger}$ Consejo Nacional de Investigaciones Científicas y Técnicas, Rivadavia 1917, C1033AAJ, Bs. As., Argentina \\ ${ }^{\top}$ Lab.Óptica, Fac. Cs. Ex. y Nat., Universidad Buenos Aires, Pab.I Cdad Universitaria, 1428 Bs.As.,Argentina \\ ' Dto Audiología Adultos, Servicio ORL, Hospital Italiano Bs. As., Gascón 450,C1181ACH, Bs.As., Argentina
}

\begin{abstract}
In Optometry and in Audiology, the routine tests to prescribe correction lenses and headsets are respectively the visual acuity test (the first chart with letters was developed by Snellen in 1862) and conventional pure tone audiometry (the first audiometer with electrical current was devised by Hartmann in 1878). At present there are psychophysical non invasive tests that, besides evaluating visual and auditory performance globally and even in cases catalogued as normal according to routine tests, supply early information regarding diseases such as diabetes, hypertension, renal failure, cardiovascular problems, etc. Concerning Optometry, one of these tests is the achromatic luminance contrast sensitivity test (introduced by Schade in 1956). Concerning Audiology, one of these tests is high frequency pure tone audiometry (introduced a few decades ago) which yields information relative to pathologies affecting the basal cochlea and complements data resulting from conventional audiometry. These utilities of the contrast sensitivity test and of pure tone audiometry derive from the facts that Fourier components constitute the basis to synthesize stimuli present at the entrance of the visual and auditory systems; that these systems responses depend on frequencies and that the patient's psychophysical state affects frequency processing. The frequency of interest in the former test is the effective spatial frequency (inverse of the angle subtended at the eye by a cycle of a sinusoidal grating and measured in cycles/degree) and, in the latter, the temporal frequency (measured in cycles/sec). Both tests have similar duration and consist in determining the patient's threshold (corresponding to the inverse multiplicative of the contrast or to the inverse additive of the sound intensity level) for each harmonic stimulus present at the system entrance (sinusoidal grating or pure tone sound). In this article the frequencies, standard normality curves and abnormal threshold shifts inherent to the contrast sensitivity test (which for simplicity could be termed "visionmetry") and to pure tone audiometry (also termed auditory sensitivity test) are analyzed with the purpose of contributing to divulge their ability to supply early information associated to pathologies not solely related to the visual and auditory systems respectively.
\end{abstract}

\section{INTRODUCTION}

The sensations of light and sound are correspondingly produced by electromagnetic and acoustic waves. In Optics and in Acoustics the medium is respectively characterized [1] by the refraction index (ratio between light propagation velocity in vacuum and in the medium) and by the specific acoustic impedance (ratio of the acoustic pressure in a medium to the associated particle velocity). Optometry and Audiology respectively deal with the way in which the eye and the ear receive and interpret magnitudes related to the electric field and fluctuations in acoustic pressure. There are both objective and subjective tests to evaluate visual and auditory performance. In this work we consider two subjective tests that are based in Fourier theory [2]; are similar to each other $[3,4]$ and measure sensitivity as a function of spatial and temporal frequency respectively, the contrast sensitivity test [3-13] and conventional and high frequency pure tone audiometry [14-22]. In Table 1 we summarize a parallelism between the eye and ear which we treat in more detail in the following sections. 


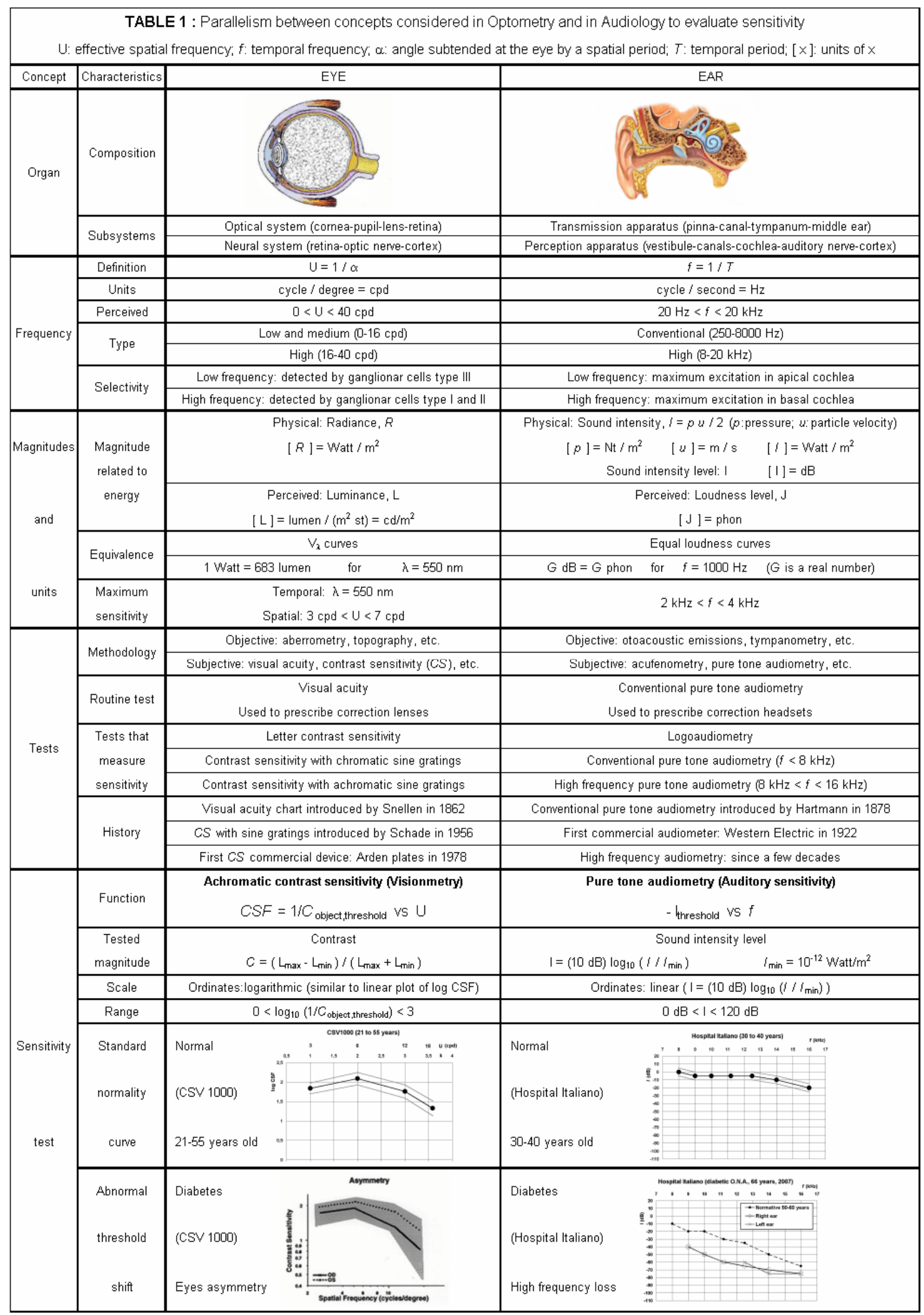




\section{OPTOMETRY AND ACHROMATIC CONTRAST SENSITIVE TEST}

In Optics there are at least 3 frequencies of interest, a temporal one related to color, a temporal one related to the interval of stimuli presentation and a spatial one related to light distribution in an object. The latter, termed $v$, is the inverse of the spatial period of a sine pattern and is measured in cycles $/ \mathrm{mm}$. When this pattern is viewed by an eye, we consider [5] the effective spatial frequency, $U=1$ cycle $/ \alpha=\nu S\left(\pi / 180^{\circ}\right)$, measured in cycles per degree, cpd, ( $\mathrm{S}$ being the distance pattern-eye and $\alpha$ the angle subtended at the eye by a period). The cutoff frequency of the visual system [5] is smaller than the cutoff frequency of the ocular optical system; it depends on pupil diameter, aberrations, accommodation, size and distribution of photoreceptors, adaptation, etc. and approximately we have [6,7] $0<\mathrm{U}<40 \mathrm{cpd}$. Low and medium frequencies $(0<\mathrm{U}<16 \mathrm{cpd})$ are detected by type III ganglion cells while high frequencies $(16<\mathrm{U}<40 \mathrm{cpd})$ are detected by types I and II ganglion cells.

In regards to energy, the eye detects luminances. The luminance, $\mathrm{L}_{\beta}$, emitted by a surface $S$ in the direction of $\beta$ ( $\beta$ being the angle between the emission direction and the normal to $S$ ) is the luminous flux, $d \Phi^{(\mathrm{L})}$, emitted by a small area projected in the direction of $\beta, d S_{Q}$, per solid angle, $d \Omega$, and per unit area, this is, $\mathrm{L}_{\beta}=d \Phi^{(\mathrm{L})} /\left(d S_{Q} d \Omega\right)$ and it is measured in lumen $/\left(\mathrm{m}^{2} \mathrm{st}\right)=\mathrm{cd} / \mathrm{m}^{2}=\mathrm{lux} / \mathrm{st}$. The eye adapts to luminances [6] in the approximate range $10^{-5} \mathrm{~cd} / \mathrm{m}^{2}<\mathrm{L}<10^{4} \mathrm{~cd} / \mathrm{m}^{2}$. A sine pattern stimulus is characterized by its mean luminance and also by its contrast, $C=\left(\mathrm{L}_{\max }-\mathrm{L}_{\min }\right) /\left(\mathrm{L}_{\max }+\mathrm{L}_{\min }\right)\left(\mathrm{L}_{\max }\right.$ and $\mathrm{L}_{\min }$ being the maximum and minimum luminances).

The most usual abnormalities of the visual system are defocus (myopia and hyperopia) and astigmatism and the routine test to prescribe correction lenses is high contrast visual acuity. The age related common anomaly is presbyopia which is the decrease in the accommodation interval. Contrast sensitivity tests detect problems not evident in the visual acuity test. There are 3 types of sensitivity tests and they are such that the targets are letters of various contrasts and sizes [7] (e.g. Pelli-Robson) and chromatic [8] and achromatic sine patterns [314] of different contrasts and frequencies. Hereby we consider tests employing achromatic sine patterns which can be displayed either on a monitor (e.g. Cambridge Research Systems VSG 2/3 [9,10] and Vistuc [5]) or on charts (e.g. Vector Vision ${ }^{\mathrm{TM}} \mathrm{CSV}-1000[4,11]$ and Vistech [3]). In these tests, the contrast sensitivity function [5-7], $C S F(\mathrm{U})=1 / C_{\text {object,threshold }}$ (where $C_{\text {object,threshold }}$ is the threshold contrast in the object), is measured for different frequencies. $C S F(\mathrm{U})$ is the visual transfer function [6] and it is the product of the modulation transfer function of the optical system, $M T F(\mathrm{U})$, and the transfer function of the neuro-retinal system, $F S P N R(\mathrm{U})$.
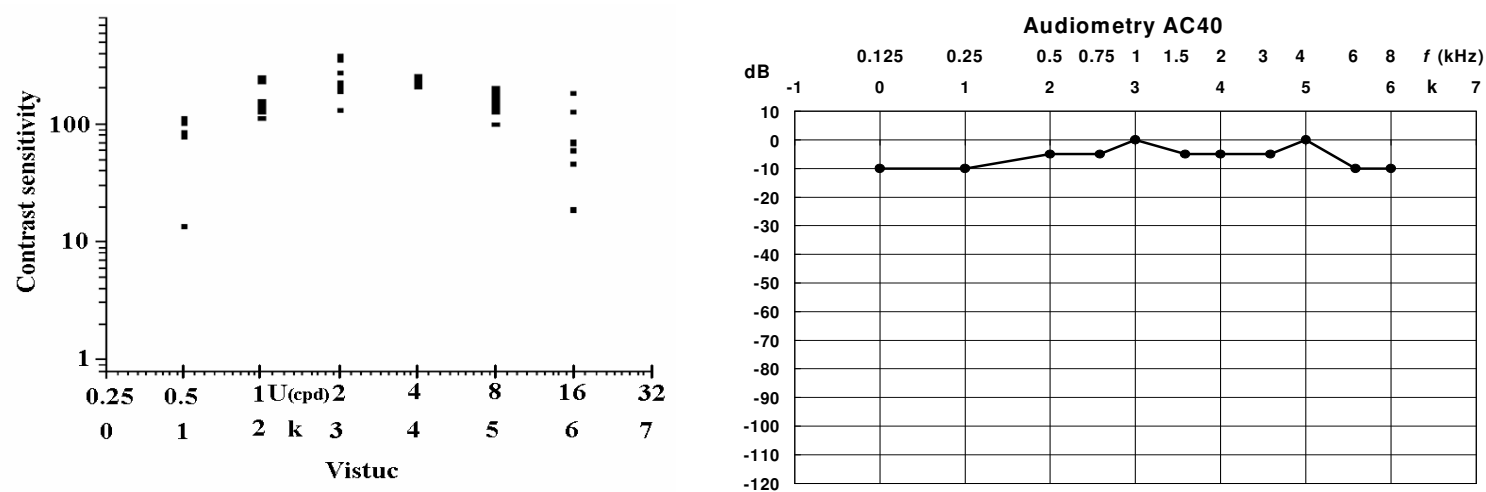

FIGURE 1. Left: $C S F$ vs $U$ (with $\mathrm{U}=\mathrm{U}_{\mathrm{o}} 2^{\mathrm{k}} ; \mathrm{U}_{\mathrm{o}}=0.25 \mathrm{cpd}$; $\mathrm{k}$ integer) obtained with the Vistuc [5] for young (20-29 years old). Right: Audiogram with $f=f_{\mathrm{o}} 2^{\mathrm{k}}$ and $f_{\mathrm{o}}=0.125 \mathrm{kHz}$ obtained with AC40 (adapted with permission of Interacoustics [20])

Concerning the plot $C S F$ vs U, log contrast sensitivity, $\log C S F=\log _{10}\left(\left(\left(\mathrm{~L}_{\max }+\mathrm{L}_{\min }\right) /\left(\mathrm{L}_{\max }-\mathrm{L}_{\min }\right)\right)_{\text {object,threshold }}\right)$ is often [3-6] plotted (Fig.1 (left)), its peak being [6] at $3 \mathrm{cpd}$ to $7 \mathrm{cpd}$. The coordinates used in the absisas varies according to various authors, the scale being linear [11], base 10 logarithmic [6-8], base 2 logarithmic [9] or corresponding [3,5] to a real number $\mathrm{k}$ such that $U=U_{\mathrm{o}} 2^{\mathrm{k}}$ (e.g. $U_{\mathrm{o}}=1.5 \mathrm{cpd}$ in the Vistech [3] while $\mathrm{U}_{\mathrm{o}}=0.25 \mathrm{cpd}$ in the Vistuc [5]). Since there are no standards accepted by the whole optical community to determine $\operatorname{CSF}(\mathrm{U})$ and/or to present results, it is necessary to dispose of normative data inherent to the device. As an example we consider the Vector Vision CSV1000E which consists [4] in a chart with 4 rows of sine 
patterns that, at the recommended distance of $2.5 \mathrm{~m}$, correspond to 4 frequencies $(3 ; 6 ; 12$ and $18 \mathrm{cpd})$ and, for each, there are 8 contrasts. In each row there are photocells that measure the total (internal plus external) luminous flux and maintain the luminance mean level constant and equal to $85 \mathrm{~cd} / \mathrm{m}^{2}$. In Fig.2 we show 2 normative plots of $\log$ CSF vs U for photopic conditions and 2 ranges of ages, 21-55 and 50-75 years old.
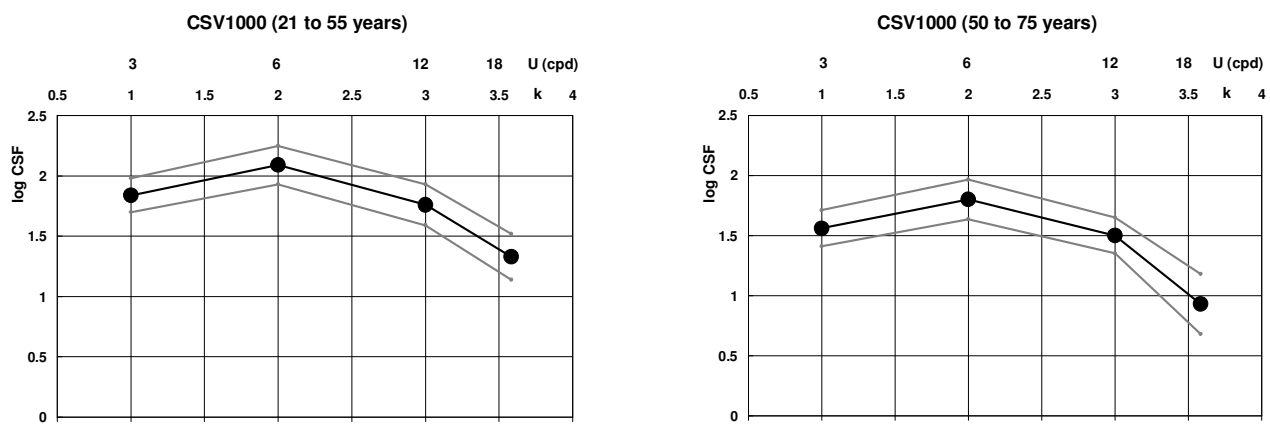

FIGURE 2. Normative plots $\log C S F$ vs $U$ (with $U=U_{0} 2^{k}$ and $U_{o}=1.5 \mathrm{cpd}$ ) under photopic conditions (left:21-55 years old, right: 50-75 years old) obtained with the CSV1000E (adapted with permission of D. Evans, President Vector Vision [4])

The contrast sensitivity test is the most accurate method of measuring sensitivity of all the visual channels [3], it informs about the ability of a subject to perceive patterns corresponding to a wide range of effective spatial frequencies and contrasts and it is a valuable tool for an early non invasive diagnose of pathologies both exclusive and inclusive of the visual system. Concerning vision, it is useful to evaluate the global visual performance of subjects that wear contact lenses [3], have intraocular implants [11], suffer from cataracts [3], underwent refractive surgery [10], suffer from macular degeneration or glaucoma [3], are aging [9], perform demanding visual tasks [12] or sports [3], etc. [5,6]. Concerning pathologies not solely affecting the visual system, it can be used to monitor pharmacological treatments [4], it supplies an early warning of diabetes [4] and multiple sclerosis [13], etc. For example, in the case of diabetes the patient's curves for both eyes can either lie below the normal range or be in this range presenting asymmetry between eyes and recent results suggest the measurement of the CSF could be useful to control insulin treatments. In the figure at the bottom of Table 1 (left) we show results [4] for a diabetic patient with no retinopathy and visual acuity 20/20.

\section{AUDIOLOGY AND CONVENTIONAL AND HIGH FREQUENCY AUDIOMETRY}

In Audiology the frequency of interest is the temporal one, $f$, measured in cycles per second $(\mathrm{Hz})$ and $f=1 / T$, $T$ being the temporal period of a harmonic sound. For example for the piano, the fundamental frequency in $\mathrm{do}_{1}$ is $264 \mathrm{~Hz}$ and in $\mathrm{do}_{\mathrm{k}+1}$ it is $264 \mathrm{~Hz} 2^{\mathrm{k}}$ (where $\mathrm{k}$ is an integer), the interval between $\mathrm{do}_{1}$ and $\mathrm{do}_{\mathrm{k}+1}$ being $\mathrm{k}$ octaves. Conventional frequencies $(20-8000 \mathrm{~Hz})$ are mapped at the apical cochlea while high frequencies (8$20 \mathrm{kHz}$ ) are mapped at the basal cochlea [14] and this map is reflected in the tonotopy of the auditory nerve.

In regards to energy, the human ear is a sound pressure sensitive detector $[1,15]$. Three magnitudes related to pressure are often considered: $p, p_{r m s}$ and $\mathrm{L}_{\mathrm{p}}$. Sound pressure, $p$, is the maximum deviation from local ambient pressure, the standard barometric pressure being $1.01310^{5} \mathrm{Nt} / \mathrm{m}^{2}$. Effective sound pressure, $p_{\text {rms }}$, is the root mean square over an interval of time of instantaneous sound pressure. Sound pressure level or sound level, $\mathrm{L}_{\mathrm{p}}=10 \log _{10}\left(p_{r m s}{ }^{2} / p_{o}{ }^{2}\right)$, is measured in $\mathrm{dBSPL}$ and $p_{\mathrm{o}}=2010^{-6} \mathrm{Nt} / \mathrm{m}^{2}$. Moreover, $p$ is related to sound intensity, $I$, by $I=p^{2} /(2 Z)=p u / 2$ ( $Z$ being the acoustic impedance and $u$ the particle velocity). Three magnitudes related to intensity are often considered: $I$, I and J. Sound intensity, $I$, is the average energy propagated through a normal area per unit area and time and is measured in Watt $/ \mathrm{m}^{2}$. Sound intensity level, $I$, ( $I \equiv \mathrm{L}_{\mathrm{p}}$ for plane progressive waves [1]) is $\mathrm{I}=10 \log _{10}\left(I / I_{\min }\right)$ measured in $\mathrm{dB}(1 \mathrm{~dB}$ being the minimum variation the ear can detect at $1 \mathrm{kHz}$ ) and $I_{\text {min }}=10^{-12} \mathrm{Watt} / \mathrm{m}^{2}$ is related to $p_{\mathrm{o}}$ and to $Z$. Sound loudness, J, is a subjective term describing the strength of the ear's perception of a sound; it is related to sound intensity factoring for each frequency according to the equal loudness curves to take into account the non flat response of the ear and is measured in phon (if $G$ is a real number and $0<G<120$ then $G \mathrm{~dB}=G$ phon at $1 \mathrm{kHz}$ ). Usually an audible sound is characterized by its sound intensity level and the ear detects without pain levels such that $0 \mathrm{~dB}<\mathrm{I}<120 \mathrm{~dB}$. 
The most usual abnormality of the auditory system is hypoacusis which can be either transmissive or sensorineural. The age related hearing loss is presbyacusis which shifts the perceived threshold sound level and decreases cutoff frequency. The routine test to diagnose the type and degree of hypoacusis and to prescribe headsets that amplify the required frequencies is conventional pure tone audiometry. In each ear, both the aerial and bone vias can be evaluated and comparing these results, the ear region causing the problem can be localized. To test the aerial via, for frequencies in the range $125 \mathrm{~Hz}<f<8000 \mathrm{~Hz}$, the threshold sound intensity level, $\mathrm{I}_{\text {threshold }}=10 \log _{10}\left(I_{\text {threshold }} / I_{\text {min }}\right.$ ), is measured (often in dBHL [20], HL indicating hearing level or hearing loss) and regarding the positive axis as pointing upwards, $-\mathrm{I}_{\text {threshold }}$ is plotted. The maximum sensitivity of human audition [1] is between $2 \mathrm{kHz}$ and $4 \mathrm{kHz}$. Besides conventional audiometry there are 2 other sensitivity tests, logoaudiometry and high frequency pure tone audiometry $(8 \mathrm{kHz}<f<16 \mathrm{kHz})$ which contributes to a better understanding of the basal cochlear damage complementing conventional audiometry.
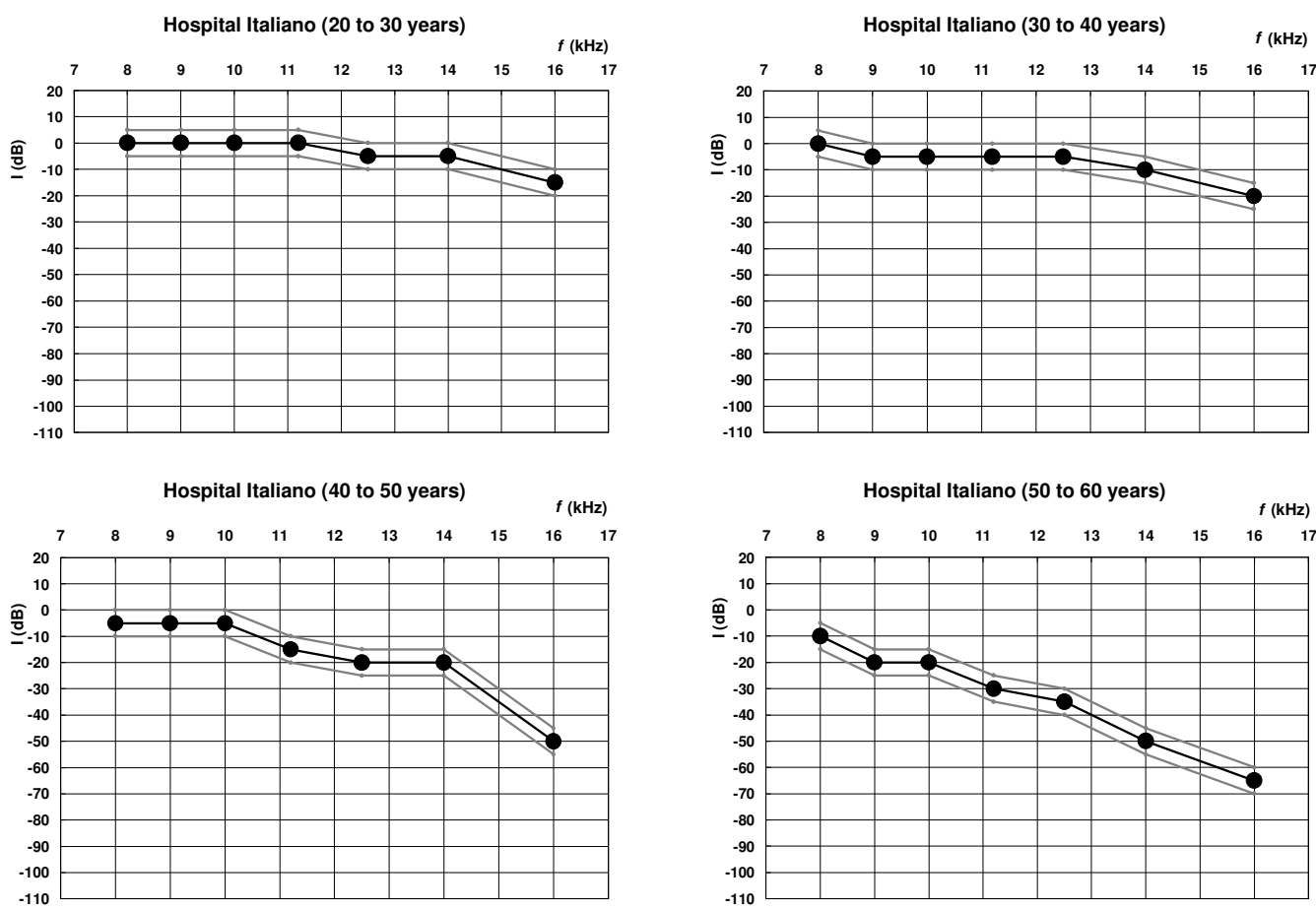

FIGURE 3. High frequency audiometries for normal hearing obtained at the Hospital Italiano of Buenos Aires [20]

Concerning the plot $-\mathrm{I}_{\text {threshold }}$ VS $f$, in conventional audiograms the frequencies often considered [16] are such that $f=f_{\mathrm{o}} 2^{k}, f_{\mathrm{o}}$ being a fixed value usually equal to $250 \mathrm{~Hz}$ and $k=\log _{2}(f)-\log _{2}\left(f_{\mathrm{o}}\right)$ a real number usually integer so that octaves are taken into account while in high frequency audiograms, $\mathrm{k}$ is not always an integer [17]. Moreover many authors do not use scientific notation in the absisas e.g. Monteiro et al [17] consider conventional and high frequencies $(0.25 ; 0.5 ; 1 ; 2 ; 3 ; 4 ; 6 ; 8 ; 10 ; 12.5 ; 16 \mathrm{kHz})$ and plot $f=f_{\mathrm{o}} 2^{\mathrm{k}}$ in a linear scale and Reis et al [18] consider high frequencies $(9 ; 10 ; 11.2 ; 12.5 ; 14 ; 16 \mathrm{kHz})$ and a linear scale. In Fig. 1 (right panel) we show an audiogram supplied in the Tutorial [19] of the audiometer AC40 of Interacoustics (which manufactures high frequency audiometers since 1986), frequencies $f=f_{\mathrm{o}} 2^{\mathrm{k}}$ (with $f_{\mathrm{o}}=0.125 \mathrm{kHz}, \mathrm{k}$ real and $0<\mathrm{k}<6$ ) are scientifically plotted. Because of the absence of accepted standards to determine $\mathrm{I}_{\text {threshold }}$ and/or to present results, it is necessary to dispose of the audiometer normative data. In 2002 Debas et al [20] determine normality curves for high frequency audiometry, the audiometer employed is the Kamplex AC 40 and the technique is mixed (from sound to silence in steps of 10dBHL and from silence to sound in steps of $5 \mathrm{dBHL}$ ). Considering 70 normal subjects of both sexes (140 ears), 4 normality curves (standard deviation being approximately 5dBHL) corresponding to 20-30, 30-40, 40-50 and 50-60 years old are obtained (Fig.3).

Conventional and high frequency audiometry are valuable tools for an early non invasive warning of pathologies both exclusive and inclusive of the auditory system. Concerning auditory anomalies, audiometry 
is useful to detect hearing impairment caused by noise and ototoxicity [14], to determine audition before and after performing various tasks [21], to evaluate the results of ears surgery, etc. Concerning pathologies not solely involving the ear, abnormal threshold shifts can be associated to hypertension, alterations in blood flux in cardiovascular surgeries, brucellosis [16], diabetes [18], chronic renal failure [22], etc. In the figure at the bottom of Table 1 (right) we show high frequency results for a diabetic insulin-dependent patient treated at the Hospital Italiano of Buenos Aires. The patient has retinopathy and bilateral sensorineural hypoacusis, in 2005 conventional audiometry revealed a $30 \mathrm{~dB}$ hearing loss in both ears at $4000 \mathrm{~Hz}$; in 2007 this loss further increased to $40 \mathrm{~dB}$ in the left ear and to $60 \mathrm{~dB}$ in the right ear and, additionally, in 2007, high frequency audiometry detected a bilateral hearing loss of more than $20 \mathrm{~dB}$ for frequencies between $9 \mathrm{kHz}$ and $14 \mathrm{kHz}$.

\section{DISCUSSION}

The achromatic contrast sensitivity test (or visionmetry) and the conventional and high frequency pure tone audiometry have almost the same duration and, even in cases in which routine tests yield normal results, they can supply an early non invasive warning of anomalies not solely affecting the visual and auditory systems. Audiometry is usually employed in the clinics, there are technicians and chambers prepared to implement it and it is known by many current citizens while the contrast sensitivity test is not so frequently used or known.

\section{ACKNOWLEDGEMENTS}

We acknowledge the support of Universidad de Buenos Aires. We are grateful to D.Evans of Vector Vision, and to A.K.Knudsen of Interacoustics for permissions to present their plots and to E.D. Carbón for the figures.

\section{REFERENCES}

1 L. E. Kinsler and A. R. Frey, Fundamentals of Acoustics, John Wiley and Sons Inc., New York, 1959.

2. J. W. Goodman, Introduction to Fourier Optics, Mc Graw-Hill, New York, 1996.

3. Vistech Consultants Inc., www.vistechconsultants.com

4. Vector Vision, http://www.vectorvision.com/html/testsContrastSensitivity.html

5. S. A. Comastri, J. M. Simon, G. Martin, E. Colombo, L. Issolio, J. Santillán and R. Aguirre, Ópt. Pura Apl. 40 (1), 119 128 (2007).

6. P. Kaufman and A. Alm, Adler fisiología del ojo, Elsevier, Madrid, 2004.

7. C. Owsley, Ophthalmol. Clin. N. Am. 16, 171-177 (2003).

8. P. Artal, S. Marcos, I. Iglesias and G. Green, Vis. Res. 36, 3575-3586 (1996).

9. Y. K. Nio, N. M. Jansonius, V. Fidler, E .Geraghty, S. Norby and A. C. Kooijman, Ophthal. Physiol. 20 (4), $323-334$ (2000).

10. J. W. W. Chan, M. H. Edwards, G. C. Woo and V. C. P. Woo, J. Cataract Refract. Surg. 28, 1774-1779 (2002).

11. E. K. Mela, S. P. Gartaganis and J. X. Koliopoulos, Documenta Ophthalmologica 92, 79-91 (1996).

12. A. P. Ginsburg, D. Evans, R. Sekuler and S. Harp, Am. J. Optom. and Physiol. Opt. 59, 105-109 (1982).

13. D. Sisto, M. Trojano, M. Vetrugno, T. Trabucco, G. Riceto and C. Sborgia, Invest. Ophthalmol. Vis. Sci. 46 (4), 12641268 (2005).

14. S. A. Fausti, V. D. Larson, D. Noffsinger, R. H. Wilson, D. S. Phillips and C.G. Fowler, Ear Hear.15, 232-239 (1994). 15. Gonzalo de Sebastián, Audiología Práctica, Editorial Médica Panamericana, Buenos Aires, 1992.

16. T. O. Kaygusuz, I. Kaygusuz, S. S. Kilic, S. Yalcin and S. Felek, Clinical Microbiology and Infection 11 (7), $559-563$ (2005).

17. I. Monteiro de Castro Silva and M. A. Guimardes Feitosa, Brazilean J. of Otorrinolaringology 72 (5), 665-672 (2006).

18. H. Goncalves Reis, B. Tschiedel and J. P. Broto, Scientia Medica Porto Alegre, PUCRS 16 (1), 16-22 (2006).

19. Interacoustics (Denmark) www.interacoustics.com

20. J. Debas, M. I. Debas, S. Dominguez, O. Borro, C. Angarano, M. E. Ben, M. T. Gargantini, M. Lanusse, F. Luzzi and M. V. Ranieri, Fonoaudiología, Asociación Argentina de Logopedia, Foniatría y Audiología 48 (2), 57-60 (2002).

21. P. Kuronen, M. J. Sorri, R. Paakkonen and A.Muhli, Int. J. Audiol. 42 (1), 29-33 (2003).

22. T. Chryssoula, T. Elias, D. Vassilis, P. Ploumis and V. Vassilis, Nephrology Dialysis Transplantation 21 (11), $3023-$ 3030 (2006). 